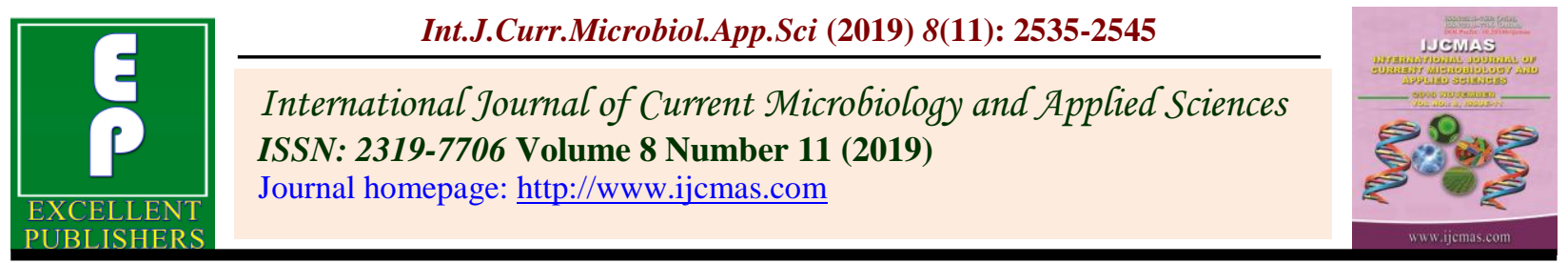

Original Research Article

https://doi.org/10.20546/ijcmas.2019.811.293

\title{
Effect of Seed Treatment with Nanoparticles on Seed Storability of Soybean
}

\author{
Dangi Sandeep $^{1 *}$, N.K. Biradarpatil ${ }^{1}$, V.K. Deshpande, Ravi Hunje ${ }^{1}$ and Suma Mogali ${ }^{2}$ \\ ${ }^{1}$ Department of Seed Science and Technology, ${ }^{2}$ Department of Genetics and Plant Breeding, \\ College of Agriculture, Dharwad-580 005, Karnataka, India \\ University of Agricultural Sciences, Dharwad, India
}

*Corresponding author

Keywords

Soybean,

Nanoparticles,

Fenps, Germination and seedling vigour index

Article Info

Accepted:

26 October 2019

Available Online:

10 November 2019

\section{A B S T R A C T}

The present storage study was carried out at Seed Quality and Research Laboratory, University of Agricultural Sciences, Dharwad during 20162017. In this study two different seed lots were taken first seed lot having higher germination percentage and second seed lot having lower germination percentage. Seeds were dry dressed with nanoparticles of silver and iron with different concentration and kept for storage. Seed lots revealed significant variation of the seed quality and storability due to nanoparticles treatments on soybean cv. DSb-21. High vigour seed lot recorded higher seed germination and seedling vigour index-I than the low vigour seed lot at the end of storage. The nanoparticles treatment FeNPs @500 ppm which recorded significantly higher seed germination $(92.5 \%)$ and seedling vigour index- I (3127) than control at the beginning, maintained the trend till the end of 10 month of storage period. The rate of reduction in quality parameters was much higher in the low vigour seed lot. The seed treatment with nanoparticles reduced the rate of deterioration of seed in the storage.

\section{Introduction}

Soybean [Glycine max (L.) Merill] is one of the most important protein and oilseed crop throughout the world. Soybean was introduced to India during 1880. Its oil is the largest component of the world's edible oils accounting for 30 per cent of the world oil production of edible oil.
Currently, it ranks first in oilseed production. Despite the high yielding potential and advantages of soybean cultivation, the yield per unit area of the crop is low in India. Poor germination and low seed viability are among the serious problems limiting the production of soybean. Soybean absorbs 50 per cent of its weight in moisture to germinate compared to only 30 per cent for maize. It must be planted 
in soils with adequate moisture to ensure maximum germination.

Research on the storability of seeds with modified storage conditions, in India is of a recent origin with the development of organized seed production and marketing. It is stipulated that 80 per cent of certified seed produced in India requires storage for one planting season and 20 per cent of seed is carried over for subsequent sowing (Bal, 1976). However, with the awareness and infrastructure development, substantial quality of seeds may be stored for few planting seasons as a safeguard against monsoon failure and as a precaution against production of poor quality seeds. The loss of seed viability due to seed deterioration is inexorable, irreversible and inevitable which is mainly dependent on physical, physiological and chemical composition of seed (Delouche et al., 1973).

However, the rate of deterioration could be slow down to a greater extent during storage by imposing certain seed treatments before storage. Use of nano-technological approaches expected to quench the reactive oxygen species and reduce the oxidative damage to improve the viability and vigour of seeds.

Metal nanoparticles are derived from salts of gold $(\mathrm{Au})$, silver $(\mathrm{Ag})$, iron $(\mathrm{Fe})$, copper $(\mathrm{Cu})$, zinc $(\mathrm{Zn})$, titanium dioxide $\left(\mathrm{TiO}_{2}\right)$, cadmium $(\mathrm{Cd})$, aluminium $(\mathrm{Al})$ and silica $(\mathrm{Si})$ etc. This study hypothesized that the soybean seeds stored for an extended period of time may result in the production of reactive oxygen species that damages the cell wall integrity as a consequence of lipid peroxidation.

Nanoparticles are known to donate electrons that pairs with the free radicals and smother the impact of these radicals. This process collectively contributes for circumventing the damages caused during seed storage, while augmenting vigour and viability. Thus the use of these nanoparticles my find a solution for shorter longevity of soybean seeds. During the past decade, some work has been done in biological system to address a wide range of field problems utilizing nano-materials and nano-devices. Natarajan and Sivasubramanian (2008) have elucidated various nanotechnological approaches that can be employed in seed science.

Keeping all these aspects in view, the present investigation entitled "Effect of seed treatment with nanoparticles on seed longevity of soybean" was carried out.

\section{Materials and Methods}

The present storage study was carried out at Seed Quality and Research laboratory, University of Agricultural Sciences, Dharwad during 2016- 2017.

Nanoparticles are dry dressed with soybean seeds of variety DSb-21 with AgNPs @ 500, 1000,1500 ppm and FeNPs@500, 1000\& $1500 \mathrm{ppm}$ at the rate of $2 \mathrm{~g}$ per $\mathrm{kg}$ were packed in polythene pouches and stored at ambient conditions at National Seed Project Laboratory, UAS Dharwad for a period of 10 months. Required initial observations were recorded before storing the samples at National Seed Project Laboratory, University of Agricultural Sciences, Dharwad under ambient condition.

The data was statistically analyzed by using analysis of variance for completely randomized design in factorial concept for storage experiment. Main and interaction effects were compared by using ' $F$ ' test at 0.01 level of probability for laboratory experiments. $\mathrm{CD}$ values were calculated and treatments were compared as per standard procedure of analysis (Steel and Torrie, 1980). 


\section{Results and Discussion}

Soybean is emerging as most versatile and economically important crop in today's farming system but, seed longevity is a major and challenging problem to be addressed. It is considered short lived as compared to other oil seed crops and its seed germination and vigour is often reduced prior to planting. Hence, the study was conducted to prolong the seed storability and maintaining higher vigour.

Nanotechnology is one of the fast developing technologies and its products are very useful in all fields, because of their small size $\left(10^{-9} \mathrm{~nm}\right)$ and large surface area. Nanoparticles offer a larger surface-to-volume ratio and a higher concentration of partially coordinated surface sites than the corresponding bulk materials. The unique properties of nanoparticles are due to a strong interplay between elastic, geometric and electronic parameters. The result of these features is often improved by physical and chemical properties compared to that of bulk material. In recent past, efforts have been made to improve agricultural yield through exhaustive research in nanotechnology. The green revolution resulted in injudicious usage of pesticides and chemical fertilizers which caused the loss of soil biodiversity.

Several intrinsic and extrinsic factors influence the viability of seeds during storage. Among intrinsic and extrinsic factors, seed moisture content, relative humidity, temperature of storage, pests and diseases and oxygen availability are more important. Deterioration of seed is a natural process which is inevitable, inexorable and irreversible but the rate of deterioration of seed may differ due to genetic factor (Robert, 1972), storage environment (Roberts, 1961), period of storage (Reddy, 1985) and seed treatment (Zhang et al., 2005) etc.
Noticeable and consistent variation in seed quality parameters were observed in the entire ten months of storage period irrespective of seed quality level and nanoparticles treatments. Final germination, root length, shoot length, seedling dry weight and seedling vigour index were significantly maximum $(73.96 \%, 16.63 \mathrm{~cm}, 12.72 \mathrm{~cm}, 1.08 \mathrm{~g}$, and 2103.68 , respectively) at the initial period but declined gradually to minimum value (43.21 $\%, 8.82 \mathrm{~cm}, 8.02 \mathrm{~cm}, 0.789 \mathrm{~g}$, and 1247.96, respectively) at the end of ten months of storage period. Whereas, the initial EC value $\left(0.530 \mathrm{dS} \mathrm{m}^{-1}\right)$ increased to $1.940 \mathrm{dS} \mathrm{m}$. Moisture content of seed (10.01, 9.93, 9.80, $9.48,8.86$ and $8.21 \%$ ) showed gradual decrease across the storage period. The marked decrease in the seed quality parameters under advancing storage period may be attributed to seed coat characters (Delouhe et al, 1973), age induced physicochemical seed deterioration, lipid peroxidation leading to production of toxic metabolites that act upon cell and cell organelles (Maguire, 1977, Tappel, 1980 and Sohal, 1987) denaturation of proteins and enzymes (Roberts, 1961).

The present study revealed that, irrespective of nanoparticles treatments, seed lots differed significantly due to seed quality throughout the ten months of storage period. Seed germination percentage, shoot length, root length, seedling length, seedling dry weight, seedling vigour index and electrical conductivity showed significant difference due to seed lots. Seeds of high vigour seed lot $\left(\mathrm{V}_{1}\right)$ recorded higher seed quality parameters $(87.32 \%, 14.05 \mathrm{~cm}, 17.45 \mathrm{~cm}, 31.5 \mathrm{~cm} 1.114$ g, 2673, respectively) over the low vigour seed lot $\left(\mathrm{V}_{2}\right)(60.61 \%, 11.39 \mathrm{~cm}, 15.81 \mathrm{~cm}$, $25.31 \mathrm{~cm}, 1.048 \mathrm{~g}, 1534$, respectively) at the initial month of storage.

The gradual reduction in these quality parameters with the increase in storage period 
was noticed in both seed lots. At the end of ten months storage higher quality parameters were recorded in seeds of high vigour seed lot $(57.54 \%, 9.40 \mathrm{~cm}, 9.64 \mathrm{~cm}, 19.040 .656 \mathrm{~g}, 742$, $31.88,1764,0.809$ respectively) as compared to the seeds of low vigour seed lot $(28.29 \%$, $6.65 \mathrm{~cm}, 7.99 \mathrm{~cm}, 14.64 \mathrm{~cm}, 594 \mathrm{~g}, 731,0.769$ $\mathrm{g}$ respectively).

Similar variation in electrical conductivity of seed leachates was observed at all the months of storage period. At initial month of storage lower electrical conductivity $\left(0.48 \mathrm{dS} \mathrm{m}^{-1}\right)$ was recorded in $\mathrm{V}_{1}$ and higher $\left(0.58 \mathrm{dS} \mathrm{m}^{-1}\right)$ was recorded in $\mathrm{V}_{2}$. At the end of ten months of storage lower electrical conductivity (1.73

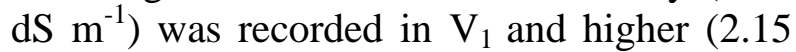
$\mathrm{dS} \mathrm{m}^{-1}$ ) was recorded in $\mathrm{V}_{2}$. Among the seed lots $\left(\mathrm{V}_{1}\right)$ high vigour seed lot maintained about 70 per cent meeting the minimum germination requirement prescribed as per
Minimum Seed Certification Standards (IMSCS) (Anon., 2013d) up to six months period, The present findings are in accordance with Vashisth and Nagarajan (2009) in Maize.

The seed quality parameters showed significant difference due to nanoparticles seed treatment. Seeds treated with FeNPs (500 ppm) $\left(\mathrm{N}_{4}\right)$ recorded the higher germination, root length, shoot length, seedling length, seedling dry weight, seedling vigour index and lower electrical conductivity $(76.38 \%, 17.02$ $\mathrm{cm}, 13.37 \mathrm{~cm}, 29.71 \mathrm{~cm}, 0.659 \mathrm{~g}, 2267,1.097$

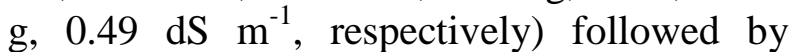
FeNPs $(500 \mathrm{ppm})\left(\mathrm{N}_{4}\right)(47.38 \%, 17.02 \mathrm{~cm}$, $13.37 \mathrm{~cm}, 29.71 \mathrm{~cm}, 1.091 \mathrm{~g}, 2251$ and 0.51

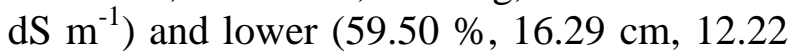
$\mathrm{cm}, 26.51 \mathrm{~cm}, 1931,1.063 \mathrm{~g}$, and $0.56 \mathrm{dS} \mathrm{m}^{-1}$, respectively) in untreated (control) $\left(\mathrm{N}_{7}\right)$ at the end of ten months storage (Table 1-5).

Table.1 Effect of seed vigour levels and nanopaticles seed treatments on germination $(\%)$ of soybean

\begin{tabular}{|c|c|c|c|c|c|c|}
\hline \multirow[t]{2}{*}{ Treatments } & \multicolumn{6}{|c|}{ Storage period (months) } \\
\hline & Initial & 2 & 4 & 6 & 8 & 10 \\
\hline \multicolumn{7}{|c|}{ Seed quality (V) } \\
\hline $\begin{array}{l}\mathbf{V}_{1} \text {-High } \\
\text { vigour }\end{array}$ & $\begin{array}{c}87.32 \\
(69.14)\end{array}$ & $\begin{array}{c}84.57 \\
(66.87)\end{array}$ & $\begin{array}{c}78.86 \\
(62.63)\end{array}$ & $\begin{array}{c}73.43 \\
(58.97)\end{array}$ & $\begin{array}{c}65.79 \\
(54.20)\end{array}$ & $\begin{array}{c}57.54 \\
(49.33)\end{array}$ \\
\hline $\begin{array}{l}V_{2} \text {-Low } \\
\text { vigour }\end{array}$ & $\begin{array}{c}60.61 \\
(51.13)\end{array}$ & $\begin{array}{c}56.79 \\
(48.90)\end{array}$ & $\begin{array}{c}52.93 \\
(46.68)\end{array}$ & $\begin{array}{c}46.18 \\
(42.81)\end{array}$ & $\begin{array}{c}37.75 \\
(37.91)\end{array}$ & $\begin{array}{c}28.89 \\
(32.52)\end{array}$ \\
\hline S. Em. \pm & 0.37 & 0.35 & 0.38 & 0.31 & 0.45 & 0.39 \\
\hline C. D. at $1 \%$ & 1.42 & 1.35 & 1.43 & 1.17 & 1.71 & 1.48 \\
\hline \multicolumn{7}{|c|}{ Nanoparticles Treatments $(\mathrm{N})$} \\
\hline $\mathbf{N}_{1}$ & $\begin{array}{c}73.63 \\
(59.10)\end{array}$ & $\begin{array}{c}70.00 \\
(56.79)\end{array}$ & $\begin{array}{c}65.75 \\
(54.18)\end{array}$ & $\begin{array}{c}60.25 \\
(50.91)\end{array}$ & $\begin{array}{c}51.75 \\
(46.00)\end{array}$ & $\begin{array}{c}43.38 \\
(41.19)\end{array}$ \\
\hline $\mathbf{N}_{2}$ & $\begin{array}{c}74.13 \\
(59.43)\end{array}$ & $\begin{array}{c}70.75 \\
(57.26)\end{array}$ & $\begin{array}{c}66.25 \\
(54.48)\end{array}$ & $\begin{array}{c}60.75 \\
(51.21)\end{array}$ & $\begin{array}{c}52.88 \\
(46.65)\end{array}$ & $\begin{array}{c}44.50 \\
(41.84)\end{array}$ \\
\hline $\mathbf{N}_{3}$ & $\begin{array}{c}72.88 \\
(58.62)\end{array}$ & $\begin{array}{c}69.63 \\
(56.56)\end{array}$ & $\begin{array}{c}64.88 \\
(53.66)\end{array}$ & $\begin{array}{c}58.75 \\
(50.04)\end{array}$ & $\begin{array}{c}50.25 \\
(45.14)\end{array}$ & $\begin{array}{c}40.88 \\
(39.74)\end{array}$ \\
\hline $\mathbf{N}_{4}$ & $\begin{array}{c}76.38 \\
(60.92)\end{array}$ & $\begin{array}{c}73.50 \\
(59.02)\end{array}$ & $\begin{array}{c}68.13 \\
(55.63)\end{array}$ & $\begin{array}{c}62.13 \\
(52.02)\end{array}$ & $\begin{array}{c}55.25 \\
(48.01)\end{array}$ & $\begin{array}{c}47.38 \\
(43.50)\end{array}$ \\
\hline $\mathbf{N}_{5}$ & $\begin{array}{c}75.00 \\
(60.00)\end{array}$ & $\begin{array}{c}72.00 \\
(58.05)\end{array}$ & $\begin{array}{c}67.13 \\
(55.02)\end{array}$ & $\begin{array}{c}61.63 \\
(51.73)\end{array}$ & $\begin{array}{c}53.88 \\
(47.23)\end{array}$ & $\begin{array}{c}45.63 \\
(42.49)\end{array}$ \\
\hline
\end{tabular}




\begin{tabular}{|c|c|c|c|c|c|c|}
\hline $\mathbf{N}_{6}$ & $\begin{array}{c}73.38 \\
(58.94)\end{array}$ & $\begin{array}{c}70.13 \\
(56.87)\end{array}$ & $\begin{array}{c}65.38 \\
(53.96)\end{array}$ & $\begin{array}{c}59.50 \\
(50.48)\end{array}$ & $\begin{array}{c}50.00 \\
(45.00)\end{array}$ & $\begin{array}{c}41.88 \\
(40.32)\end{array}$ \\
\hline $\mathbf{N}_{7}$ & $\begin{array}{c}72.38 \\
(58.29)\end{array}$ & $\begin{array}{c}68.75 \\
(56.01)\end{array}$ & $\begin{array}{c}63.75 \\
(52.98)\end{array}$ & $\begin{array}{c}55.63 \\
(48.23)\end{array}$ & $\begin{array}{c}48.38 \\
(44.07)\end{array}$ & $\begin{array}{c}38.88 \\
(38.57)\end{array}$ \\
\hline S. Em. \pm & 0.70 & 0.66 & 0.70 & 0.57 & 0.84 & 0.72 \\
\hline C.D. at $1 \%$ & 2.67 & 2.52 & 2.68 & 2.18 & 3.20 & 2.77 \\
\hline \multicolumn{7}{|c|}{ Interaction $(\mathbf{V} \times \mathbf{N})$} \\
\hline $\mathbf{V}_{1} \mathbf{N}_{1}$ & $\begin{array}{c}86.75 \\
(68.65)\end{array}$ & $\begin{array}{c}83.50 \\
(66.03)\end{array}$ & $\begin{array}{c}78.50 \\
(62.38)\end{array}$ & $\begin{array}{c}74.00 \\
(59.34)\end{array}$ & $\begin{array}{c}67.00 \\
(54.94)\end{array}$ & $\begin{array}{c}58.00 \\
(49.60)\end{array}$ \\
\hline $\mathbf{V}_{1} \mathbf{N}_{2}$ & $\begin{array}{c}87.50 \\
(69.30)\end{array}$ & $\begin{array}{c}84.50 \\
(66.82)\end{array}$ & $\begin{array}{c}79.00 \\
(62.73)\end{array}$ & $\begin{array}{c}74.50 \\
(59.67)\end{array}$ & $\begin{array}{c}68.00 \\
(55.55)\end{array}$ & $\begin{array}{c}59.50 \\
(50.48)\end{array}$ \\
\hline $\mathrm{V}_{1} \mathbf{N 3}$ & $\begin{array}{c}85.75 \\
(67.82)\end{array}$ & $\begin{array}{c}83.25 \\
(65.84)\end{array}$ & $\begin{array}{c}77.75 \\
(61.86)\end{array}$ & $\begin{array}{c}72.50 \\
(58.37)\end{array}$ & $\begin{array}{c}62.50 \\
(52.24)\end{array}$ & $\begin{array}{c}54.50 \\
(47.58)\end{array}$ \\
\hline $\mathbf{V}_{1} \mathbf{N}_{4}$ & $\begin{array}{c}90.75 \\
(72.29)\end{array}$ & $\begin{array}{c}88.50 \\
(70.18)\end{array}$ & $\begin{array}{c}81.25 \\
(64.34)\end{array}$ & $\begin{array}{c}76.75 \\
(61.17)\end{array}$ & $\begin{array}{c}70.00 \\
(56.79)\end{array}$ & $\begin{array}{c}62.75 \\
(52.39)\end{array}$ \\
\hline $\mathbf{V}_{1} \mathbf{N}_{5}$ & $\begin{array}{c}88.75 \\
(70.40)\end{array}$ & $\begin{array}{c}86.25 \\
(68.23)\end{array}$ & $\begin{array}{c}80.00 \\
(63.43)\end{array}$ & $\begin{array}{c}76.00 \\
(60.67)\end{array}$ & $\begin{array}{c}69.50 \\
(56.48)\end{array}$ & $\begin{array}{c}60.50 \\
(51.06)\end{array}$ \\
\hline $\mathbf{V}_{1} \mathbf{N}_{6}$ & $\begin{array}{c}86.50 \\
(68.44)\end{array}$ & $\begin{array}{c}84.00 \\
(66.42)\end{array}$ & $\begin{array}{c}78.25 \\
(62.20)\end{array}$ & $\begin{array}{c}73.25 \\
(58.86)\end{array}$ & $\begin{array}{c}63.25 \\
(52.68)\end{array}$ & $\begin{array}{c}56.25 \\
(48.59)\end{array}$ \\
\hline $\mathbf{V}_{1} \mathbf{N}_{7}$ & $\begin{array}{c}85.25 \\
(67.41)\end{array}$ & $\begin{array}{c}82.00 \\
(64.90)\end{array}$ & $\begin{array}{c}77.25 \\
(61.51)\end{array}$ & $\begin{array}{c}67.00 \\
(54.94)\end{array}$ & $\begin{array}{c}60.25 \\
(50.91)\end{array}$ & $\begin{array}{c}51.25 \\
(45.72)\end{array}$ \\
\hline $\mathbf{V}_{2} \mathbf{N}_{1}$ & $\begin{array}{c}60.50 \\
(51.06)\end{array}$ & $\begin{array}{c}56.50 \\
(48.73)\end{array}$ & $\begin{array}{c}53.00 \\
(46.72)\end{array}$ & $\begin{array}{c}46.50 \\
(42.99)\end{array}$ & $\begin{array}{c}36.50 \\
(37.17)\end{array}$ & $\begin{array}{c}28.75 \\
(32.42)\end{array}$ \\
\hline $\mathbf{V}_{2} \mathbf{N}_{2}$ & $\begin{array}{c}60.75 \\
(51.21)\end{array}$ & $\begin{array}{c}57.00 \\
(49.02)\end{array}$ & $\begin{array}{c}53.50 \\
(47.01)\end{array}$ & $\begin{array}{c}47.00 \\
(43.28)\end{array}$ & $\begin{array}{c}37.75 \\
(37.91)\end{array}$ & $\begin{array}{c}29.50 \\
(32.90)\end{array}$ \\
\hline $\mathbf{V}_{2} \mathbf{N}_{3}$ & $\begin{array}{c}60.00 \\
(50.77)\end{array}$ & $\begin{array}{c}56.00 \\
(48.45)\end{array}$ & $\begin{array}{c}52.00 \\
(46.15)\end{array}$ & $\begin{array}{c}45.00 \\
(42.13)\end{array}$ & $\begin{array}{c}38.00 \\
(38.06)\end{array}$ & $\begin{array}{c}27.25 \\
(31.47)\end{array}$ \\
\hline $\mathbf{V}_{2} \mathbf{N}_{4}$ & $\begin{array}{c}62.00 \\
(51.94)\end{array}$ & $\begin{array}{c}58.50 \\
(49.89)\end{array}$ & $\begin{array}{c}55.00 \\
(47.87)\end{array}$ & $\begin{array}{c}47.50 \\
(43.57)\end{array}$ & $\begin{array}{c}40.50 \\
(39.52)\end{array}$ & $\begin{array}{c}32.00 \\
(34.45)\end{array}$ \\
\hline $\mathbf{V}_{2} \mathbf{N}_{5}$ & $\begin{array}{c}61.25 \\
(51.50)\end{array}$ & $\begin{array}{c}57.75 \\
(49.46)\end{array}$ & $\begin{array}{c}54.25 \\
(47.44)\end{array}$ & $\begin{array}{c}47.25 \\
(43.42)\end{array}$ & $\begin{array}{c}38.25 \\
(38.20)\end{array}$ & $\begin{array}{c}30.75 \\
(33.68)\end{array}$ \\
\hline $\mathbf{V}_{2} \mathbf{N}_{6}$ & $\begin{array}{c}60.25 \\
(50.91)\end{array}$ & $\begin{array}{c}56.25 \\
(48.59)\end{array}$ & $\begin{array}{c}52.50 \\
(46.43)\end{array}$ & $\begin{array}{c}45.75 \\
(42.56)\end{array}$ & $\begin{array}{c}36.75 \\
(37.32)\end{array}$ & $\begin{array}{c}27.50 \\
(31.63)\end{array}$ \\
\hline $\mathbf{V}_{2} \mathbf{N}_{7}$ & $\begin{array}{c}59.50 \\
(50.48)\end{array}$ & $\begin{array}{c}55.50 \\
(48.16)\end{array}$ & $\begin{array}{c}50.25 \\
(45.14)\end{array}$ & $\begin{array}{r}44.25 \\
(41.70)\end{array}$ & $\begin{array}{c}36.50 \\
(37.17)\end{array}$ & $\begin{array}{c}26.50 \\
(30.98)\end{array}$ \\
\hline Mean & $\begin{array}{c}73.96 \\
(59.32)\end{array}$ & $\begin{array}{c}70.68 \\
(\mathbf{5 7 . 2 2})\end{array}$ & $\begin{array}{c}65.89 \\
(54.26)\end{array}$ & $\begin{array}{c}\mathbf{5 9 . 8 0} \\
(\mathbf{5 0 . 6 5})\end{array}$ & $\begin{array}{c}51.77 \\
(46.01)\end{array}$ & $\begin{array}{c}43.21 \\
(41.10)\end{array}$ \\
\hline S. Em. \pm & 0.97 & 0.94 & 0.99 & 0.81 & 1.19 & 1.02 \\
\hline C. D. at $1 \%$ & NS & NS & NS & 3.08 & 4.53 & NS \\
\hline
\end{tabular}

Figures in parentheses indicate arc sine values

$\mathrm{V}_{1}=$ Seed lot- 1 (Seed lot with high vigour $85 \%$ germination), $\mathrm{V}_{2}=$ Seed lot- 2 (Seed lot with low vigour, 55\% germination)

$\mathrm{N}_{1}=$ TiNPs @500 ppm, $\mathrm{N}_{2}=$ TiNPs @ 1000 ppm, $\mathrm{N}_{3}=$ TiNPs @ 1500 ppm, $\mathrm{N}_{4=}$ ZnNPs @ $500 \mathrm{ppm}, \mathrm{N}_{5}=$ ZnNPs @ 1000 ppm, $\mathrm{N}_{6}=$ ZnNPs @ 1500 ppm, $\mathrm{N}_{7}=$ without nanoparticle treatment (control) 
Table.2 Effect of seed vigour levels and nanopaticles seed treatments on seedling vigour index-I of soybean

\begin{tabular}{|c|c|c|c|c|c|c|}
\hline \multirow[t]{2}{*}{ Treatments } & \multicolumn{6}{|c|}{ Storage period(months) } \\
\hline & Initial & 2 & 4 & 6 & 8 & 10 \\
\hline \multicolumn{7}{|l|}{ Seed quality (V) } \\
\hline $\begin{array}{l}\mathrm{V}_{1} \text {-High } \\
\text { vigour }\end{array}$ & 2673 & 2589 & 2413 & 2249 & 2017 & 1768 \\
\hline $\mathrm{V}_{2}$-Low vigour & 1534 & 1437 & 1340 & 1170 & 955 & 732 \\
\hline S. Em. \pm & 20.17 & 16.11 & 15.82 & 14.19 & 16.22 & 13.81 \\
\hline C. D. at $1 \%$ & 76.96 & 61.46 & 60.37 & 54.15 & 61.89 & 52.68 \\
\hline \multicolumn{7}{|c|}{ Priming treatments $(\mathbf{N})$} \\
\hline $\mathbf{N}_{1}$ & 2078 & 1989 & 1854 & 1693 & 1428 & 1203 \\
\hline $\mathbf{N}_{2}$ & 2135 & 2041 & 1911 & 1759 & 1538 & 1301 \\
\hline $\mathbf{N}_{3}$ & 1999 & 1913 & 1784 & 1620 & 1387 & 1137 \\
\hline $\mathbf{N}_{4}$ & 2267 & 2179 & 2031 & 1871 & 1645 & 1413 \\
\hline $\mathbf{N}_{5}$ & 2252 & 2169 & 2007 & 1840 & 1640 & 1412 \\
\hline $\mathbf{N}_{6}$ & 2062 & 1963 & 1845 & 1696 & 1465 & 1233 \\
\hline $\mathbf{N}_{7}($ control $)$ & 1932 & 1836 & 1704 & 1486 & 1298 & 1049 \\
\hline S. Em. \pm & 37.73 & 30.13 & 29.60 & 26.55 & 30.34 & 25.83 \\
\hline C. D. at $1 \%$ & 143.97 & 114.98 & 112.94 & 101.31 & 115.78 & 98.55 \\
\hline \multicolumn{7}{|c|}{ Interaction $(\mathbf{V} \times \mathbf{N})$} \\
\hline $\mathbf{V}_{1} \mathbf{N}_{1}$ & 2634 & 2558 & 2382 & 2230 & 1933 & 1712 \\
\hline $\mathbf{V}_{1} \mathbf{N}_{2}$ & 2713 & 2619 & 2449 & 2309 & 2108 & 1845 \\
\hline $\mathrm{V}_{1} \mathbf{N 3}$ & 2538 & 2462 & 2299 & 2143 & 1847 & 1610 \\
\hline $\mathbf{V}_{1} \mathbf{N}_{4}$ & 2891 & 2809 & 2605 & 2474 & 2263 & 2000 \\
\hline $\mathbf{V}_{1} \mathbf{N}_{5}$ & 2887 & 2814 & 2583 & 2441 & 2227 & 1993 \\
\hline $\mathbf{V}_{1} \mathbf{N}_{6}$ & 2620 & 2520 & 2370 & 2234 & 2022 & 1752 \\
\hline $\mathbf{V}_{1} \mathbf{N}_{7}$ & 2430 & 2338 & 2203 & 1909 & 1719 & 1463 \\
\hline $\mathbf{V}_{2} \mathbf{N}_{1}$ & 1523 & 1421 & 1326 & 1157 & 923 & 694 \\
\hline $\mathbf{V}_{2} \mathbf{N}_{2}$ & 1556 & 1462 & 1372 & 1208 & 968 & 757 \\
\hline $\mathbf{V}_{2} \mathbf{N}_{3}$ & 1461 & 1364 & 1268 & 1097 & 927 & 664 \\
\hline $\mathbf{V}_{2} \mathbf{N}_{4}$ & 1644 & 1550 & 1456 & 1268 & 1027 & 825 \\
\hline $\mathrm{V}_{2} \mathrm{~N}_{5}$ & 1616 & 1524 & 1432 & 1238 & 1054 & 831 \\
\hline $\mathbf{V}_{2} \mathbf{N}_{6}$ & 1504 & 1406 & 1319 & 1159. & 908 & 715 \\
\hline $\mathrm{V}_{2} \mathrm{~N}_{7}$ & 1433 & 1334 & 1206 & 1063 & 877 & 635 \\
\hline Mean & 2103.68 & 2012.98 & 1876.62 & 1709.36 & 1486.00 & 1249.93 \\
\hline S. Em. \pm & 53.36 & 42.62 & 41.86 & 37.55 & 42.91 & 36.53 \\
\hline C. D. at $1 \%$ & NS & NS & NS & 143.28 & 163.74 & 139.37 \\
\hline
\end{tabular}

$\mathrm{V}_{1}=$ Seed lot-1 (Seed lot with high vigour $85 \%$ germination)

$\mathrm{V}_{2}=$ Seed lot-2 (Seed lot with low vigour, 55\% germination)

$\mathrm{N}_{1}=$ AgNPs @ 500 ppm, $\mathrm{N}_{2}=$ AgNPs @ 1000 ppm, $\mathrm{N}_{3}=$ AgNPs @ 1500 ppm, $\mathrm{N}_{4=}$ FeNPs @ 500 ppm, $\mathrm{N}_{5}=$ FeNPs @ 1000 ppm, $N_{6}=$ FeNPs @ 1500 ppm, $N_{7}=$ without nanoparticle treatment (control) 
Table.3 Effect of seed vigour levels and nanopaticles seed treatments on seedling vigour index-II of soybean.

\begin{tabular}{|c|c|c|c|c|c|c|}
\hline \multirow[t]{2}{*}{ Treatments } & \multicolumn{6}{|c|}{ Storage period(months) } \\
\hline & Initial & 2 & 4 & 6 & 8 & 10 \\
\hline \multicolumn{7}{|l|}{ Seed quality (V) } \\
\hline $\begin{array}{l}V_{1} \text {-High } \\
\text { vigour }\end{array}$ & 97.27 & 90.32 & 80.45 & 71.94 & 59.17 & 46.57 \\
\hline $\mathrm{V}_{2}$-Low vigour & 63.49 & 57.54 & 51.91 & 42.60 & 34.55 & 22.13 \\
\hline S. Em. \pm & 0.18 & 0.11 & 0.14 & 0.10 & 0.11 & 0.11 \\
\hline C. D. at $1 \%$ & 0.68 & 0.43 & 0.52 & 0.36 & 0.42 & 0.43 \\
\hline \multicolumn{7}{|c|}{ Priming treatments $(\mathbf{N})$} \\
\hline $\mathbf{N}_{1}$ & 80.00 & 73.55 & 66.13 & 57.88 & 47.49 & 34.30 \\
\hline $\mathbf{N}_{2}$ & 80.80 & 74.52 & 67.04 & 58.52 & 48.58 & 35.36 \\
\hline $\mathbf{N}_{3}$ & 78.47 & 72.17 & 64.25 & 55.58 & 45.42 & 31.78 \\
\hline $\mathbf{N}_{4}$ & 84.25 & 78.47 & 69.79 & 60.80 & 51.56 & 38.34 \\
\hline $\mathbf{N}_{5}$ & 82.25 & 76.37 & 68.39 & 59.92 & 49.99 & 36.60 \\
\hline $\mathbf{N}_{6}$ & 79.48 & 72.98 & 65.09 & 56.81 & 43.65 & 34.31 \\
\hline $\mathbf{N}_{7}($ control $)$ & 77.41 & 69.46 & 62.56 & 51.40 & 41.32 & 29.79 \\
\hline S. Em. \pm & 80.00 & 0.21 & 0.25 & 0.18 & 0.20 & 0.21 \\
\hline C. D. at $1 \%$ & 80.80 & 0.81 & 0.97 & 0.68 & 0.78 & 0.80 \\
\hline \multicolumn{7}{|c|}{ Interaction $(\mathbf{V} \times \mathbf{N})$} \\
\hline $\mathbf{V}_{1} \mathbf{N}_{1}$ & 96.52 & 89.53 & 80.03 & 72.54 & 60.34 & 47.00 \\
\hline $\mathbf{V}_{1} \mathbf{N}_{2}$ & 97.84 & 90.66 & 80.82 & 73.39 & 61.35 & 48.36 \\
\hline $\mathrm{V}_{1} \mathbf{N 3}$ & 94.80 & 87.99 & 78.20 & 70.09 & 55.34 & 43.35 \\
\hline $\mathbf{V}_{1} \mathbf{N}_{4}$ & 102.28 & 96.28 & 84.25 & 76.76 & 64.32 & 51.99 \\
\hline $\mathbf{V}_{1} \mathbf{N}_{5}$ & 99.59 & 93.20 & 82.58 & 75.35 & 63.34 & 49.76 \\
\hline$V_{1} N_{6}$ & 96.09 & 89.21 & 79.24 & 71.30 & 56.60 & 45.14 \\
\hline $\mathbf{V}_{1} \mathbf{N}_{7}$ & 93.76 & 85.38 & 78.02 & 64.15 & 52.91 & 40.41 \\
\hline $\mathbf{V}_{2} \mathbf{N}_{1}$ & 63.48 & 57.57 & 52.24 & 43.22 & 34.65 & 21.60 \\
\hline $\mathbf{V}_{2} \mathbf{N}_{2}$ & 63.75 & 58.39 & 53.26 & 43.64 & 35.81 & 22.36 \\
\hline $\mathbf{V}_{2} \mathbf{N}_{3}$ & 62.13 & 56.36 & 50.30 & 41.06 & 35.51 & 20.21 \\
\hline $\mathbf{V}_{2} \mathbf{N}_{4}$ & 66.23 & 60.67 & 55.33 & 44.84 & 38.80 & 24.70 \\
\hline $\mathrm{V}_{2} \mathrm{~N}_{5}$ & 64.92 & 59.54 & 54.19 & 44.48 & 36.64 & 23.43 \\
\hline $\mathrm{V}_{2} \mathrm{~N}_{6}$ & 62.87 & 56.76 & 50.94 & 42.32 & 30.70 & 23.48 \\
\hline $\mathrm{V}_{2} \mathrm{~N}_{7}$ & 61.07 & 53.54 & 47.09 & 38.64 & 29.73 & 19.17 \\
\hline Mean & 80.38 & 73.93 & 66.18 & 57.27 & 46.86 & 34.35 \\
\hline S. Em. \pm & 0.47 & 0.30 & 0.36 & 0.25 & 0.29 & 0.29 \\
\hline C. D. at $1 \%$ & 1.81 & 1.14 & 1.37 & 0.96 & 1.10 & 1.13 \\
\hline
\end{tabular}

$\mathrm{V}_{1}=$ Seed lot-1 (Seed lot with high vigour $85 \%$ germination)

$\mathrm{V}_{2}=$ Seed lot-2 (Seed lot with low vigour, $55 \%$ germination)

$\mathrm{N}_{1}=$ AgNPs @ 500 ppm, $\mathrm{N}_{2}=$ AgNPs @ 1000 ppm, $\mathrm{N}_{3}=$ AgNPs @ 1500 ppm, $\mathrm{N}_{4=}$ FeNPs @ 500 ppm, $\mathrm{N}_{5}=$ FeNPs @ 1000 ppm, $\mathrm{N}_{6}=$ FeNPs @ 1500 ppm, $\mathrm{N}_{7}=$ without nanoparticle treatment (control) 
Table.4 Effect of seed vigour levels and nanopaticles seed treatments on seedling dry weight (g) of soybean ( $\mathrm{g} / 10$ seedlings)

\begin{tabular}{|c|c|c|c|c|c|c|}
\hline \multirow[t]{2}{*}{ Treatments } & \multicolumn{6}{|c|}{ Storage period(months) } \\
\hline & Initial & 2 & 4 & 6 & 8 & 10 \\
\hline \multicolumn{7}{|l|}{ Seed quality (V) } \\
\hline$V_{1}$-High vigour & 1.114 & 1.068 & 1.020 & 0.979 & 0.899 & 0.809 \\
\hline $\mathrm{V}_{2}$-Low vigour & 1.048 & 1.013 & 0.979 & 0.925 & 0.917 & 0.769 \\
\hline S. Em. \pm & 0.002 & 0.001 & 0.003 & 0.003 & 0.003 & 0.003 \\
\hline C. D. at $1 \%$ & 0.006 & 0.005 & 0.010 & 0.013 & 0.012 & 0.010 \\
\hline \multicolumn{7}{|c|}{ Nanoparticles treatments $(\mathbf{N})$} \\
\hline $\mathbf{N}_{1}$ & 1.081 & 1.044 & 1.003 & 0.954 & 0.925 & 0.784 \\
\hline $\mathbf{N}_{2}$ & 1.087 & 1.049 & 1.009 & 0.958 & 0.927 & 0.787 \\
\hline $\mathbf{N}_{3}$ & 1.071 & 1.031 & 0.985 & 0.939 & 0.910 & 0.769 \\
\hline $\mathbf{N}_{4}$ & 1.097 & 1.063 & 1.022 & 0.974 & 0.942 & 0.802 \\
\hline $\mathbf{N}_{5}$ & 1.091 & 1.056 & 1.015 & 0.967 & 0.936 & 0.797 \\
\hline $\mathbf{N}_{6}$ & 1.076 & 1.036 & 0.992 & 0.948 & 0.868 & 0.828 \\
\hline $\mathbf{N}_{7}($ control $)$ & 1.063 & 1.004 & 0.973 & 0.926 & 0.847 & 0.757 \\
\hline S. Em. \pm & 0.003 & 0.002 & 0.005 & 0.006 & 0.006 & 0.005 \\
\hline C. D. at $1 \%$ & 0.011 & 0.009 & 0.019 & 0.025 & 0.023 & 0.020 \\
\hline \multicolumn{7}{|c|}{ Interaction $(\mathbf{V} \times \mathbf{N})$} \\
\hline $\mathbf{V}_{1} \mathbf{N}_{1}$ & 1.114 & 1.071 & 1.020 & 0.980 & 0.901 & 0.810 \\
\hline $\mathbf{V}_{1} \mathbf{N}_{2}$ & 1.119 & 1.073 & 1.024 & 0.984 & 0.903 & 0.814 \\
\hline $\mathrm{V}_{1} \mathbf{N 3}$ & 1.106 & 1.057 & 1.006 & 0.966 & 0.886 & 0.796 \\
\hline $\mathbf{V}_{1} \mathbf{N}_{4}$ & 1.127 & 1.088 & 1.037 & 1.002 & 0.918 & 0.828 \\
\hline $\mathrm{V}_{1} \mathrm{~N}_{5}$ & 1.122 & 1.081 & 1.033 & 0.992 & 0.912 & 0.822 \\
\hline $\mathrm{V}_{1} \mathbf{N}_{6}$ & 1.110 & 1.062 & 1.013 & 0.972 & 0.892 & 0.802 \\
\hline $\mathbf{V}_{1} \mathbf{N}_{7}$ & 1.100 & 1.043 & 1.011 & 0.958 & 0.879 & 0.789 \\
\hline $\mathrm{V}_{2} \mathrm{~N}_{1}$ & 1.048 & 1.018 & 0.986 & 0.927 & 0.949 & 0.757 \\
\hline $\mathbf{V}_{2} \mathbf{N}_{2}$ & 1.055 & 1.025 & 0.994 & 0.932 & 0.952 & 0.760 \\
\hline $\mathbf{V}_{2} \mathbf{N}_{3}$ & 1.035 & 1.005 & 0.964 & 0.912 & 0.934 & 0.742 \\
\hline $\mathbf{V}_{2} \mathbf{N}_{4}$ & 1.068 & 1.038 & 1.007 & 0.945 & 0.965 & 0.775 \\
\hline $\mathrm{V}_{2} \mathrm{~N}_{5}$ & 1.061 & 1.031 & 0.998 & 0.942 & 0.961 & 0.771 \\
\hline $\mathrm{V}_{2} \mathrm{~N}_{6}$ & 1.042 & 1.011 & 0.970 & 0.924 & 0.844 & 0.853 \\
\hline $\mathrm{V}_{2} \mathrm{~N}_{7}$ & 1.026 & 0.966 & 0.936 & 0.895 & 0.815 & 0.725 \\
\hline Mean & 1.08 & 1.04 & 1.00 & 0.952 & 0.908 & 0.789 \\
\hline S. Em. \pm & 0.004 & 0.003 & 0.007 & 0.009 & 0.009 & 0.007 \\
\hline C. D. at $1 \%$ & NS & 0.012 & NS & NS & 0.033 & 0.028 \\
\hline
\end{tabular}

$\mathrm{V}_{1}=$ Seed lot-1 (Seed lot with high vigour $85 \%$ germination)

$\mathrm{V}_{2}=$ Seed lot-2 (Seed lot with low vigour, 55\% germination)

$\mathrm{N}_{1}=$ AgNPs@500 ppm, $\mathrm{N}_{2}=$ AgNPs@1000 ppm, $\mathrm{N}_{3}=$ AgNPs@1500 ppm, $\mathrm{N}_{4=}$ FeNPs@500 ppm, $\mathrm{N}_{5}=$ FeNPs @ 1000 ppm, $\mathrm{N}_{6}=$ FeNPs @ 1500 ppm, $\mathrm{N}_{7}=$ without nanoparticle treatment (control) 
Table.5 Effect of seed vigour levels and nanopaticles seed treatments on electrical conductivity $\left(\mathrm{dS}^{-1} \mathrm{~m}\right)$ of soybean

\begin{tabular}{|c|c|c|c|c|c|c|}
\hline \multirow[t]{2}{*}{ Treatments } & \multicolumn{6}{|c|}{ Storage period(months) } \\
\hline & Initial & 2 & 4 & 6 & 8 & 10 \\
\hline \multicolumn{7}{|l|}{ Seed quality (V) } \\
\hline $\mathrm{V}_{1}$-High vigour & 0.48 & 0.57 & 0.71 & 1.11 & 1.47 & 1.73 \\
\hline $\mathrm{V}_{2}$-Low vigour & 0.58 & 0.68 & 0.80 & 1.26 & 1.68 & 2.15 \\
\hline S. Em. \pm & 0.00 & 0.01 & 0.01 & 0.01 & 0.01 & 0.02 \\
\hline C. D. at $1 \%$ & 0.02 & $\mathbf{0 . 0 2}$ & 0.02 & 0.04 & $\mathbf{0 . 0 3}$ & 0.07 \\
\hline \multicolumn{7}{|c|}{ Nanoparticles treatments $(\mathbf{N})$} \\
\hline $\mathbf{N}_{1}$ & 0.53 & 0.63 & 0.76 & 1.18 & 1.57 & 1.91 \\
\hline $\mathbf{N}_{2}$ & 0.52 & 0.62 & 0.74 & 1.16 & 1.55 & 1.84 \\
\hline $\mathbf{N}_{3}$ & 0.55 & 0.65 & 0.78 & 1.22 & 1.67 & 2.07 \\
\hline $\mathbf{N}_{4}$ & 0.49 & 0.59 & 0.72 & 1.13 & 1.46 & 1.81 \\
\hline $\mathbf{N}_{5}$ & 0.51 & 0.60 & 0.73 & 1.15 & 1.48 & 1.82 \\
\hline $\mathbf{N}_{6}$ & 0.55 & 0.64 & 0.77 & 1.20 & 1.61 & 1.97 \\
\hline $\mathbf{N}_{7}($ control $)$ & 0.56 & 0.66 & 0.79 & 1.24 & 1.69 & 2.16 \\
\hline S. Em. \pm & 0.01 & 0.01 & 0.01 & 0.02 & 0.01 & 0.04 \\
\hline C. D. at $1 \%$ & 0.03 & 0.05 & 0.04 & 0.08 & 0.06 & 0.14 \\
\hline \multicolumn{7}{|c|}{ Interaction $(\mathrm{V} \times \mathbf{N})$} \\
\hline $\mathbf{V}_{1} \mathbf{N}_{1}$ & 0.48 & 0.57 & 0.71 & 1.11 & 1.46 & 1.69 \\
\hline $\mathbf{V}_{1} \mathbf{N}_{2}$ & 0.46 & 0.56 & 0.69 & 1.09 & 1.46 & 1.58 \\
\hline $\mathrm{V}_{1} \mathrm{N3}$ & 0.51 & 0.60 & 0.74 & 1.16 & 1.62 & 1.96 \\
\hline $\mathbf{V}_{1} \mathbf{N}_{4}$ & 0.43 & 0.53 & 0.67 & 1.05 & 1.30 & 1.55 \\
\hline $\mathbf{V}_{1} \mathbf{N}_{5}$ & 0.45 & 0.55 & 0.68 & 1.07 & 1.32 & 1.56 \\
\hline $\mathrm{V}_{1} \mathrm{~N}_{6}$ & 0.51 & 0.59 & 0.72 & 1.13 & 1.54 & 1.81 \\
\hline $\mathbf{V}_{1} \mathbf{N}_{7}$ & 0.53 & 0.61 & 0.74 & 1.18 & 1.62 & 1.97 \\
\hline $\mathbf{V}_{2} \mathbf{N}_{1}$ & 0.58 & 0.68 & 0.81 & 1.25 & 1.67 & 2.13 \\
\hline $\mathbf{V}_{2} \mathbf{N}_{2}$ & 0.57 & 0.67 & 0.79 & 1.24 & 1.64 & 2.10 \\
\hline $\mathbf{V}_{2} \mathbf{N}_{3}$ & 0.59 & 0.70 & 0.82 & 1.28 & 1.73 & 2.18 \\
\hline $\mathbf{V}_{2} \mathbf{N}_{4}$ & 0.56 & 0.66 & 0.77 & 1.22 & 1.63 & 2.08 \\
\hline $\mathbf{V}_{2} \mathbf{N}_{5}$ & 0.57 & 0.66 & 0.78 & 1.23 & 1.64 & 2.08 \\
\hline $\mathbf{V}_{2} \mathbf{N}_{6}$ & 0.59 & 0.69 & 0.81 & 1.28 & 1.68 & 2.13 \\
\hline $\mathbf{V}_{2} \mathbf{N}_{7}$ & 0.59 & 0.71 & 0.83 & 1.30 & 1.75 & 2.35 \\
\hline Mean & $\mathbf{0 . 5 3 0}$ & 0.711 & 0.756 & 1.184 & 1.575 & 1.940 \\
\hline S. Em. \pm & 0.01 & 0.02 & 0.02 & 0.03 & 0.02 & 0.05 \\
\hline C. D. at $1 \%$ & NS & NS & NS & NS & 0.08 & NS \\
\hline
\end{tabular}

$\mathrm{V}_{1}=$ Seed lot -1 (Seed lot with high vigour $85 \%$ germination)

$\mathrm{V}_{2}=$ Seed lot -2 (Seed lot with low vigour, $55 \%$ germination)

$\mathrm{N}_{1}=$ AgNPs @ 500 ppm, $\mathrm{N}_{2}=$ AgNPs @ 1000 ppm, $\mathrm{N}_{3}=$ AgNPs @ 1500 ppm, N $4=$ FeNPs @ 500 ppm, N $5=$ FeNPs @ 1000 ppm, $\mathrm{N}_{6}=$ FeNPs $@ 1500$ ppm, $\mathrm{N}_{7}=$ without nanoparticle treatment (control)

A gradual reduction in seed quality parameters were observed with the increase in storage period was noticed across all treatments. From the FeNPs 500ppm $\left(\mathrm{N}_{4}\right)(70.2 \%)$ treated seeds maintained above 70 per cent germination percentage meeting the minimum germination requirement prescribed as per Minimum Seed Certification Standards (IMSCS) (Anon., 2013d) up to four months. However it's physiological and mechanical 
mechanisms are still poorly understood with respect to storage potentiality of seed.

In this present investigation to enhanced storability in nanoparticles seed treatment may be due possible resonance as a phenomenon which enhances seed germination performance and vigour. Further, nanoparticles seed treatment reduced conductivity of leachate through rearranging the membrane content and leadings to increase the water absorption and ionic currents in the seeds. The present findings are in accordance with Vashisth and Nagarajan (2009) in maize and reported that treating of seed to nanoparticles seed treatment reversed the extend of the deterioration caused by ageing as attributed by higher germination per cent, seedling length and seedling dry weight. Similar observation was also made by Girishet al. (2011) in cotton and Anon, (2013b) in pea.

The probable reason for the enhanced germination due to the nano size of particles allow them to penetrate through seed coat easily and hence, provided better absorption and utilization of these particles by seeds. The beneficial effect of the these NPs in improving the germination and production of essential biomolecules as well as essential nutrients required for plant growth and are important components of various enzymes which are responsible for driving many metabolic reaction (Senthilkumar, 2011).

Interaction effect between seed lots (V) and nanoparticles $(\mathrm{N})$ seed treatment $(\mathrm{V} \times \mathrm{N})$ was found to be non-significant for all the seed quality parameters studied throughout storage period of ten months. However, decrease in seed quality parameters except electrical conductivity values with increase in storage period was noticed in all the treatment combination.

In general higher seed germination (90.75\%), root length $(17.85 \mathrm{~cm})$, shoot length (14.64 $\mathrm{cm})$, seedling length $(32.49 \mathrm{~cm})$, seedling dry weight $(1.127 \mathrm{~g})$, seedling vigour index (2890), lower electrical conductivity ( $0.43 \mathrm{dS}$ $\mathrm{m}^{-1}$ ) were seen in the treatment combination of high vigour seed lot with FeNP500 ppm $\left(\mathrm{V}_{1} \mathrm{~N}_{4}\right)$ compared to untreated low vigour seed lot $\left(\mathrm{V}_{2} \mathrm{~N}_{7}\right)(59.50 \%, 15.39 \mathrm{~cm}, 11.06 \mathrm{~cm}$, $26.45 \mathrm{~cm} 1.026 \mathrm{~g}, 1433.03$, and $0.59 \mathrm{dS} \mathrm{m}^{-1}$ ) at the end of ten months of storage period.

From the FeNP 500 ppm $\left(\mathrm{N}_{4}\right)(70 \%)$ treated seeds maintained above 70 per cent germination percentage meeting the minimum germination requirement prescribed as per Indian Minimum Seed Certification Standards (IMSCS) (Anon., 2013d) up to eight months.

\section{References}

Anonymous 2013a, Soybean outlook.www. seaofindia. com. pp. 1-13

Anonymous, 2013b, Indian minimum seed certification standards. The Central Seed Certification Board, Department of Agriculture and Co-operation, Ministry of Agriculture, Government of India, New Delhi, pp. 129-130

Bal, S. S., 1976, Magnitude and types of seed storage needs in India. Seed Res., 4: 15.

BalaRaju, B. and Rai, P. K., 2017, Studies on effect of polymer seed coating, nanoparticles and hydro priming on seedling characters of pigeonpea (Cajanus cajan L.) seed. J. Pharma. Phytochem., 6 (4): 140-145

Delouche, J. C., Matter, R. K., Dougherty, G. M. and Boyde, A. H., 1973, Storage of seeds in tropical regions.Seed Sci. Technol., 1: 671-700.

Girish, K., Yadav, S. K., Lal, S. K., and Jain, S. K., 2011, Static magnetic field treatment enhances seed performance of cotton (Gossypium hirsutum L.). Seed Res., 39(2): 124-129.

Khodakovskaya, M., Dervishi, E., Mahmood, M., Xu., Li, Z. and Watanabe, F., 
2009, Carbon nanotubes are able to penetrate plant seed coat and dramatically affect seed germination and plant growth. ACS Nano.,3 (10): 3221-3227.

Leiderer, P. and Dekorsy, T., 2008, Interactions of nanoparticles and surfaces tag der maundlichen praufung. URL: http: //www. ub. unikonstanz. de/kops/ volltexte/2008/5387/; URN: http: //nbn-resolving. de/urn: nbn: de: bsz: 352-opus-53877.

Reddy, S. A., 1985, Studies on seed viability and vigour in cultivars of Gossypium hirsutumL. and G. barbadense L., M. Sc. (Agri) Thesis. Tamil Nadu Agric. Uni., Coimbatore. Tamil Nadu (India).

Roberts, E. H. and Wittington, 1972, Storage environment and control of viability. Via.Seeds. (Ed.). pp. 14-18.

Roberts, E. H., 1961, Viability of rice seed in relation to temperature, moisture content and gaseous environment. Ann. Bot., 25: 381-390.

Maguire, J. D., 1977, Seed quality and

\section{How to cite this article:}

Dangi Sandeep, N.K. Biradarpatil, V.K. Deshpande, Ravi Hunje and Suma Mogali. 2019. Effect of Seed Treatment with Nanoparticles on Seed Storability of Soybean. Int.J.Curr.Microbiol.App.Sci. 8(11): 2535-2545. doi: https://doi.org/10.20546/ijcmas.2019.811.293 germination. In: The physiology and biochemistry of seed dormancy and germination, Horht Holland Publishing Co., New York, pp. 219-235.

Senthilkumar, S., 2011, Customizing nanoparticles for the maintenance of seed vigour and viability in blackgram (Vigna mungo) cv. VBN 4. M. Sc. (Agri.) Thesis, Tamil Nadu Agricultural University, Coimbatore.

Sohal, R. S., 1987, The free radical theory of ageing: a critique. Rev. Biol. Res. Ageing, 3: 431-499.

Tappel, A. L., 1980, Vitamin E and selenium protection from in vivo lipid peroxidation. Ann. New York Acad. Sci., 335: 18.

Vashisth, A. and Nagarajan, S., 2009, Germination characteristics of seeds of maize (Zea mays L.) exposed to magnetic fields under accelerated ageing condition. J. Agric. Phys., 9: 50-58. 